\title{
Fixed point iterations for Prešić-Kannan nonexpansive mappings in product convex metric spaces
}

\author{
Hafiz Fukhar-ud-din \\ Department of Mathematics and \\ Statistics, King Fahd University of \\ Petroleum and Minerals, Saudi Arabia \\ email: hfdin@kfupm.edu.sa; \\ hfdin@yahoo.com
}

\author{
Vasile Berinde \\ Department of Mathematics and \\ Computer Science, North University \\ Center of Baia Mare (Technical \\ University of Cluj-Napoca), Romania \\ Department of Mathematics and \\ Statistics, King Fahd University of \\ Petroleum and Minerals, Saudi Arabia \\ email: vberinde@cunbm.utcluj.ro; \\ vberinde@kfupm.edu.sa
}

\begin{abstract}
We introduce Prešić-Kannan nonexpansive mappings on the product spaces and show that they have a unique fixed point in uniformly convex metric spaces. Moreover, we approximate this fixed point by Mann iterations. Our results are new in the literature and are valid in Hilbert spaces, CAT(0) spaces and Banach spaces simultaneously.
\end{abstract}

\section{Introduction and preliminaries}

Let $(X, d)$ be a metric space and $F: X \rightarrow X$ be a mapping. Then $F$ is called nonexpansive if

$$
d(F x, F y) \leq d(x, y)
$$

2010 Mathematics Subject Classification: Primary 47H09, 47H10; Secondary 49M05 Key words and phrases: convex metric space, Prešić Kannan nonexpansive mapping, fixed point, Mann iterative algorithm, convergence 
for all $x, y \in X$.

$\mathrm{F}$ is said to be Kannan nonexpansive provided that

$$
d(F x, F y) \leq \frac{1}{2}[d(x, F x)+d(y, F y)]
$$

for all $x, y \in X$.

Nonexpansive mappings are always continuous but Kannan nonexpansive mappings are discontinuous in general, see [10]. Therefore, conditions (1) and (2) are independent, that is, there exists a nonexpansive mapping which is not Kannan nonexpansive and a Kannan nonexpansive which is not nonexpansive. So, we cannot compare both the mappings directly. It is well known that nonexpansive and Kannan nonexpansive mappings on a nonempty, compact and convex subset $C$ of a Banach space $X$, have a fixed point [13]. For a weakly compact and convex subset $C$ of a Banach space $X$, the existence of fixed points for nonexpansive and Kannan nonexpansive mappings cannot be obtained. This fact was studied by Alspach[1] who showed that there is a weakly compact and convex subset $\mathrm{C}$ of $\mathrm{L}^{1}[0,1]$ such that $\mathrm{F}: \mathrm{C} \rightarrow \mathrm{C}$ is a nonexpansive mapping without a fixed point.

In 1973, Kannan [9] proved that if $\mathbf{X}$ is a reflexive Banach space and for any convex F-invariant subset $\mathrm{H}$ of $\mathrm{C}$ - nonempty bounded, closed and convex subset of $\mathrm{X}$ - which has more than one point and

$$
\sup _{y \in H}\|y-F y\|<\operatorname{diam}(H),
$$

then the Kannan nonexpansive mapping F : C $\rightarrow$ C has a fixed point. After that Soardi [24] proved the existence of a fixed point for the Kannan nonexpansive mapping $\mathrm{F}: \mathrm{C} \rightarrow \mathrm{C}$ by using the notion of normal structure and obtained a similar result to Kirk's fixed point theorem for Kannan nonexpansive mappings.

For two related but distinct concepts of nonexpansive bivariate mappings, see [3].

A convex structure [25] in a metric space $X$ is a mapping $W: X^{2} \times I \rightarrow X$ satisfying:

$$
d(u, W(x, y, \alpha)) \leq \alpha d(u, x)+(1-\alpha) d(u, y)
$$

for all $u, x, y \in X$ and $\alpha \in I=[0,1]$. In general, $W$ is not continuous but in this paper we shall assume that $W$ is continuous. Hadamard manifolds [5] and geodesic spaces [4] are the nonlinear examples of a convex metric space, while Hilbert spaces and Banach spaces are the linear ones. 
A convex metric space $X$ is uniformly convex [23] if for any $\varepsilon>0$, there exists $\delta(\varepsilon)>0$ such that $\mathrm{d}\left(z, W\left(x, y, \frac{1}{2}\right)\right) \leq \mathrm{r}(1-\delta(\varepsilon))<\mathrm{r}$ for all $\mathrm{r}>0$ and $x, y, z \in X$ with $d(z, x) \leq r, d(z, y) \leq r$ and $d(x, y) \geq r \varepsilon$.

Uniformly convex Banach spaces and CAT(0) spaces are nice examples of a uniformly convex metric space[11].

A convex metric space $X$ satisfy Property $(C)$ if

$$
W(x, y, \lambda)=W(y, x, 1-\lambda)
$$

and Property $(\mathrm{H})$ if

$$
d(W(x, y, \lambda), W(x, z, \lambda)) \leq(1-\lambda) d(y, z)
$$

for all $x, y, z \in X$ and $\lambda \in(0,1)$.

It is shown in [19] that Property $(\mathrm{C})$ and $(\mathrm{H})$ together imply continuity of $W$ and Property $(\mathrm{C})$ holds in uniformly convex metric spaces.

Recently, Fukhar et. al [7] has proved the following result:

Theorem 1 Let $\mathrm{C}$ be a nonempty, closed, convex and bounded subset of a complete and uniformly convex metric space $\mathrm{X}$ satisfying Property $(\mathrm{H})$. If $\mathrm{F}$ : $\mathrm{C} \rightarrow \mathrm{C}$ is a continuous mapping satisfying

$$
d(F x, F y) \leq a_{1} d(x, y)+a_{2} d(F x, x)+a_{3} d(F y, y)+a_{4} d(F x, y)+a_{5} d(F y, x)
$$

for all $\mathrm{x}, \mathrm{y} \in \mathrm{C}$, where $\mathrm{a}_{\mathrm{i}} \geq 0$ and $\sum_{i=1}^{5} \mathrm{a}_{\mathrm{i}} \leq 1$, then $\mathrm{F}$ has a fixed point in $\mathrm{C}$.

An interesting generalization of Banach contraction principle has been obtained by Prešić [20]:

Theorem 2 Let $(\mathrm{X}, \mathrm{d})$ be a complete metric space, $\mathrm{k}$ a positive integer, $\alpha_{1}, \alpha_{2}, \alpha_{3}, \ldots, \alpha_{k} \in \mathbb{R}_{+}, \sum_{i=1}^{k} \alpha_{i}=\alpha<1$ and $f: X^{k} \rightarrow X$ a mapping satisfying

$$
d\left(f\left(x_{0}, x_{1}, \ldots, x_{k-1}\right), f\left(x_{1}, x_{2}, \ldots, x_{k}\right)\right) \leq \sum_{i=1}^{k} \alpha_{i} d\left(x_{i-1}, x_{i}\right)
$$

for all $\mathrm{x}_{0}, \mathrm{x}_{1}, \ldots, \mathrm{x}_{\mathrm{k}} \in \mathrm{X}$. Then $\mathrm{f}$ has a unique fixed point $\mathrm{x}^{*}$, that is, there exists a unique $\mathrm{x}^{*} \in \mathrm{X}$ such that $\mathrm{f}\left(\mathrm{x}^{*}, \mathrm{x}^{*}, \ldots, \mathrm{x}^{*}\right)=\mathrm{x}^{*}$ and the sequence defined by

$$
x_{n+1}=f\left(x_{n-k+1}, \ldots, x_{n}\right), \quad n=k-1, k, k+1, \ldots
$$

converges to $\mathrm{x}^{*}$ for any $\mathrm{x}_{0}, \mathrm{x}_{1}, \ldots, \mathrm{x}_{\mathrm{k}-1} \in \mathrm{X}$. 
Some generalizations of Theorem 2 have been obtained in $[6,21]$ (see also $[15,16,17,18])$.

Recall the definition of a Prešić nonexpansive mapping, first introduced in [2].

A mapping $f: X^{k} \rightarrow X$ is a Prešić nonexpansive if

$$
d\left(f\left(x_{0}, x_{1}, \ldots, x_{k-1}\right), f\left(x_{1}, x_{2}, \ldots, x_{k}\right)\right) \leq \sum_{i=1}^{k} \alpha_{i} d\left(x_{i-1}, x_{i}\right)
$$

for all $x_{0}, x_{1}, \ldots, x_{k} \in X$, where $k$ is a positive integer, and $\alpha_{1}, \alpha_{2}, \alpha_{3}, \ldots, \alpha_{k} \in$ $\mathbb{R}_{+}, \alpha_{1}+\alpha_{2} \leq 1$.

Since in the definition of Prešić nonexpansive mapping, the constant $\alpha \leq 1$, therefore this class of mappings includes the class of Prešić contractions appearing in Theorem 2. In the case $k=1$, the Prešić nonexpansiveness condition reduces to Banach contractive condition if $\alpha<1$ and to the nonexpansiveness condition if $\alpha=1$.

A fixed point result about Prešić nonexpansive mappings in a nonlinear domain, namely, CAT(0) spaces has been obtained in [8] in the form of the following result:

Theorem 3 Let $\mathrm{C}$ be a bounded, closed and convex subset of a complete $\mathrm{CAT}(0)$ space $\mathrm{X}, \mathrm{k}$ a positive integer, and let $\mathrm{f}: \mathrm{X}^{\mathrm{k}} \rightarrow \mathrm{X}$ be a Prešić nonexpansive mapping. Then $\mathrm{f}$ has a fixed point, that is, there exists $\mathrm{X}^{*} \in \mathrm{X}$ such that $f\left(\chi^{*}, \chi^{*}, \ldots, x^{*}\right)=\chi^{*}$.

We note that for $k=1$, Theorem 3 becomes Kirk fixed point theorem in $[12]$.

These and similar facts have motivated us to study the generalization of Kannan nonexpansive mappings in product metric spaces and product convex metric spaces.

A mapping $f: X^{k} \rightarrow X$ is a Prešić Kannan nonexpansive if

$$
d\left(f\left(x_{0}, x_{1}, \ldots, x_{k-1}\right), f\left(x_{1}, x_{2}, \ldots, x_{k}\right)\right) \leq \frac{1}{k(k+1)} \sum_{i=0}^{k} d\left(x_{i}, f\left(x_{i}, x_{i}, \ldots, x_{i}\right)\right)
$$

for all $x_{0}, x_{1}, \ldots, x_{k} \in X$.

For $k=1$, it reduces to the classical Kannan nonexpansive mapping.

Example 1 Let $\mathrm{I}=[0,1]$ be the unit interval with the usual Euclidean norm and let $\mathrm{f}: \mathrm{I}^{2} \rightarrow \mathrm{I}$ be given by 


$$
f(x, y)=\left\{\begin{array}{l}
\frac{1}{6} \text { if } x<\frac{3}{4}, y \in I \\
\frac{1}{15} \text { if } x \geq \frac{3}{4}, y \in I .
\end{array}\right.
$$

Then $f$ is a Prešić Kannan nonexpansive but not Prešić nonexpansive.

Proof. In this particular case, Prešić kannan nonexpansive condition becomes:

$$
\left|f\left(x_{0}, x_{1}\right)-f\left(x_{1}, x_{2}\right)\right| \leq \frac{1}{6}\left(\begin{array}{c}
\left|x_{0}-f\left(x_{0}, x_{0}\right)\right|+\left|x_{1}-f\left(x_{1}, x_{1}\right)\right| \\
+\left|x_{2}-f\left(x_{2}, x_{2}\right)\right|
\end{array}\right)
$$

for any $x_{0}, x_{1}, x_{2} \in I$.

The way of defining $f$, we write $I^{2}=\cup_{i=1}^{4} I_{i}$ where

$$
\begin{aligned}
& I_{1}=\left\{(x, y): 0 \leq x, y \leq \frac{3}{4}\right\}, I_{2}=\left\{(x, y): \frac{3}{4} \leq x \leq 1,0 \leq y \leq \frac{3}{4}\right\} \\
& I_{3}=\left\{(x, y): \frac{3}{4} \leq x, y \leq 1\right\}, I_{4}=\left\{(x, y): 0 \leq x \leq \frac{3}{4}, \frac{3}{4} \leq y \leq 1\right\} .
\end{aligned}
$$

Now we discuss five cases.

Case I: $\left(x_{0}, x_{1}\right) \in I_{1}$ or $\left(x_{0}, x_{1}\right) \in I_{3}$ and $x_{2} \in$ I.Then $f\left(x_{0}, x_{1}\right)=f\left(x_{1}, x_{2}\right)$.

Consequently, inequality $(3)$ holds for any $x_{0}, x_{1}, x_{2}$ in the specified domain of $f$.

Case II: $\left(x_{0}, x_{1}\right) \in I_{2}, x_{2}<\frac{3}{4}$. Then $f\left(x_{0}, x_{0}\right)=\frac{1}{15}=f\left(x_{0}, x_{1}\right), f\left(x_{1}, x_{1}\right)=$ $f\left(x_{1}, x_{2}\right)=f\left(x_{2}, x_{2}\right)=\frac{1}{6}$.

Thus the condition(3) becomes:

$$
\frac{1}{10} \leq \frac{1}{6}\left(\left|x_{0}-\frac{1}{15}\right|+\left|x_{1}-\frac{1}{6}\right|+\left|x_{2}-\frac{1}{6}\right|\right)
$$

but $\frac{3}{4} \leq x_{0} \leq 1$ implies $\frac{41}{60} \leq x_{0}-\frac{1}{15} \leq \frac{14}{15}$ implies $\left|x_{0}-\frac{1}{15}\right| \geq \frac{41}{60} ; 0 \leq x_{1} \leq \frac{3}{4}$ implies $-\frac{1}{6} \leq x_{1}-\frac{1}{6} \leq \frac{7}{12}$ implies $\left|x_{1}-\frac{1}{6}\right| \geq \frac{1}{6} ; 0 \leq x_{2}<\frac{3}{4}$ implies $-\frac{1}{6} \leq$ $x_{1}-\frac{1}{6}<\frac{7}{12}$ implies $\left|x_{1}-\frac{1}{6}\right| \geq \frac{1}{6}$.

It follows that $\frac{1}{10} \leq \frac{1}{6}\left(\frac{41}{60}+\frac{2}{3}\right) \leq \frac{1}{6}\left(\left|x_{0}-\frac{1}{15}\right|+\left|x_{1}-\frac{1}{6}\right|+\left|x_{2}-\frac{1}{6}\right|\right)$ holds.

Case III: $\left(x_{0}, x_{1}\right) \in I_{2}, x_{2} \geq \frac{3}{4}$. Then $f\left(x_{0}, x_{0}\right)=f\left(x_{0}, x_{1}\right)=f\left(x_{2}, x_{2}\right)=$ $\frac{1}{15}, f\left(x_{1}, x_{1}\right)=f\left(x_{1}, x_{2}\right)=\frac{1}{6}$.

Thus the condition(3) becomes:

$$
\frac{1}{10} \leq \frac{1}{6}\left(\left|x_{0}-\frac{1}{15}\right|+\left|x_{1}-\frac{1}{6}\right|+\left|x_{2}-\frac{1}{15}\right|\right) \text {. }
$$

But $\left|x_{0}-\frac{1}{15}\right| \geq \frac{41}{60} ;\left|x_{1}-\frac{1}{6}\right| \geq \frac{1}{6} ; \frac{3}{4} \leq x_{2} \leq 1$ implies $\frac{41}{60} \leq x_{1}-\frac{1}{15}<\frac{14}{15}$ implies $\left|x_{1}-\frac{1}{15}\right| \geq \frac{41}{60}$. 
It follows that $\frac{1}{10} \leq \frac{1}{6}\left(\frac{41}{30}+\frac{1}{6}\right) \leq \frac{1}{6}\left(\left|x_{0}-\frac{1}{15}\right|+\left|x_{1}-\frac{1}{6}\right|+\left|x_{2}-\frac{1}{6}\right|\right)$ holds.

Case IV: $\left(x_{0}, x_{1}\right) \in \mathrm{I}_{4}, x_{2}<\frac{3}{4}$ and Case V: $\left(x_{0}, x_{1}\right) \in \mathrm{I}_{4}, x_{2} \geq \frac{3}{4}$ follow similarly.

Now we show that $f$ is not Prešić nonexpansive. For the above defined $f$, the Prešić nonexpansive condition becomes:

$$
\left|f\left(x_{0}, x_{1}\right)-f\left(x_{1}, x_{2}\right)\right| \leq \alpha_{1}\left|x_{0}-x_{1}\right|+\alpha_{1}\left|x_{1}-x_{2}\right|
$$

where $\alpha_{1}, \alpha_{2} \in \mathbb{R}_{+}, \alpha_{1}+\alpha_{2} \leq 1$.

Set $x_{0}=\frac{3}{4}, x_{1}=\frac{7}{10}=x_{2}, f\left(x_{0}, x_{1}\right)=\frac{1}{15}, f\left(x_{1}, x_{2}\right)=\frac{1}{6}$ in (4), we get

$$
\frac{1}{10} \leq \alpha_{1} \frac{1}{20} \Longleftrightarrow 2 \leq \alpha_{1} \leq 1
$$

a contradiction.

\section{Main results}

Kannan [9] proved the following result:

Theorem 4 Let $\mathrm{X}$ be a compact metric space and let $\mathrm{F}: \mathrm{X} \rightarrow \mathrm{X}$ be a continuous Kannan nonexpansive mapping satisfying the property that for every closed subset $\mathrm{C}$ of $\mathrm{X}$ which contains more than one point in $\mathrm{C}$ and $\mathrm{F}$ maps $C$ into itself. If there exists $\mathrm{x} \in \mathrm{C}$ such that $\mathrm{d}(\mathrm{x}, \mathrm{F}(\mathrm{x}))<\sup _{\mathrm{y} \in \mathrm{C}} \mathrm{d}(\mathrm{y}, \mathrm{F}(\mathrm{y}))$, then $\mathrm{F}$ has a unique fixed point in $\mathrm{X}$.

We extend Theorem 4 for Prešić-Kannan nonexpansive mappings on the product of compact metric spaces.

Theorem 5 Let $(\mathrm{X}, \mathrm{d})$ be a compact metric space and let $\mathrm{f}: \mathrm{X}^{\mathrm{k}} \rightarrow \mathrm{X}$ be a continuous Prešić Kannan nonexpansive mapping satisfying the property that for every closed subset $\mathrm{C}$ of $\mathrm{X}$ which contains more than one point in $\mathrm{C}$ and $\mathrm{f}\left(\mathrm{C}^{\mathrm{k}}\right) \subseteq \mathrm{C}$, there exists $\mathrm{x} \in \mathrm{C}$ such that $\mathrm{d}(\mathrm{x}, \mathrm{f}(\mathrm{x}, \mathrm{x}, \ldots, \mathrm{x}))<\sup _{\mathrm{y} \in \mathrm{C}} \mathrm{d}(\mathrm{y}$, $\mathrm{f}(\mathrm{y}, \mathrm{y}, \ldots, \mathrm{y}))$. Then $\mathrm{f}$ has a unique fixed point $\mathrm{x}^{*}$ in $\mathrm{X}$, that is, $\mathrm{f}\left(\mathrm{x}^{*}, \mathrm{x}^{*}, \ldots, \mathrm{x}^{*}\right)=$ $x^{*}$.

Proof. Define F : C $\rightarrow$ C by

$$
\mathrm{F}(z)=\mathrm{f}(z, z, \ldots, z), \quad z \in \mathrm{C} .
$$


For any $x, y \in C$, we have

$$
\begin{aligned}
& d(F(x), F(y))=d(f(x, x, \ldots, x), f(y, y, \ldots, y)) \\
& \leq d(f(x, x, \ldots, x), f(x, \ldots, x, y))+d(f(x, \ldots, x, y), f(x, \ldots, x, y, y)) \\
& +\ldots+d(f(x, y, \ldots, y), f(y, y, \ldots, y)) \\
& \leq \frac{1}{k(k+1)}[\underbrace{d(x, f(x, x, \ldots, x))+\ldots+d(x, f(x, x, \ldots, x))}_{+d(y, f(y, y, \ldots, y))}(k \text { times })] \\
& +\frac{1}{k(k+1)}[\underbrace{(2 \text { times })}_{+\underbrace{d(y, f(y, y, \ldots, y))+d(y, f(y, y, \ldots, y)})}] \\
& +\ldots \\
& +\frac{1}{k(k+1)}[+\underbrace{d(x, f(x, x, \ldots, x))}(k-1 \text { times })] \\
& =\frac{1}{k(k+1)}\left[\begin{array}{c}
d(x, F(x))(k+\ldots+2+1) \\
+d(y, F(y))(1+2+\ldots+k)
\end{array}\right] \\
& =\frac{1}{k(k+1)} \cdot \frac{k(k+1)}{2}[d(x, F(x))+d(y, F(y))] \\
& =\frac{1}{2}[d(x, F(x))+d(y, F(y))] \text {. }
\end{aligned}
$$

This shows that $F$ is a Kannan nonexpansive mapping and the conclusion follows immediately from Theorem 4 .

Theorem 6 If, in addition to the hypotheses of Theorem 5, we have

$$
d\left(f\left(x_{1}, x_{2}, \ldots, x_{k}\right), x^{*}\right) \leq \sum_{i=1}^{k} \alpha_{i} d\left(x_{i}, x^{*}\right)
$$

for all $x_{1}, x_{2}, \ldots, x_{k} \in X$ and $\alpha_{1}, \alpha_{2}, \alpha_{3}, \ldots, \alpha_{k} \in \mathbb{R}_{+}$with $x_{i} \neq x^{*}$ (a unique fixed point of $f)$ and $\sum_{i=1}^{k} \alpha_{i}<1$. Then, for every $x \in X, f^{n}(x, x, \ldots, x) \rightarrow x^{*}$.

Proof. Define $F: X \rightarrow X$ by

$$
\mathrm{F}(z)=\mathrm{f}(z, z, \ldots, z), \quad z \in \mathrm{C} .
$$


For any $x \in X$ such that $x \neq x^{*}=F\left(x^{*}\right)$, we have

$$
\begin{aligned}
d\left(F(x), x^{*}\right)= & d\left(f(x, x, \ldots, x), x^{*}\right) \\
\leq & d\left(f(x, x, \ldots, x), f\left(x, \ldots, x, x^{*}\right)\right) \\
& +d\left(f\left(x, \ldots, x, x^{*}\right), f\left(x, \ldots, x, x^{*}, x^{*}\right)\right) \\
& +\ldots+d\left(f\left(x, x^{*}, \ldots, x^{*}\right), x^{*}\right) \\
\leq & \alpha_{k} d\left(x, x^{*}\right)+\alpha_{k-1} d\left(x, x^{*}\right)+\ldots+\alpha_{1} d\left(x, x^{*}\right) \\
= & \sum_{i=1}^{k} \alpha_{i} d\left(x, x^{*}\right) \\
= & \alpha d\left(x, x^{*}\right) \\
< & d\left(x, x^{*}\right) .
\end{aligned}
$$

That is,

$$
d\left(F(x), x^{*}\right)<d\left(x, x^{*}\right) .
$$

Since $X$ is compact, there exists a subsequence $\left\{\mathrm{F}^{n_{i}}(x)\right\}$ of $\left\{F^{n}(x)\right\}$ such that $\mathrm{F}^{\mathrm{n}_{\mathrm{i}}}(\mathrm{x}) \rightarrow z \in \mathrm{X}$.

Note that

$$
\begin{aligned}
d\left(F^{n}(x), x^{*}\right) & =d\left(F^{n}(x), F\left(x^{*}\right)\right) \\
& \leq \frac{1}{2}\left\{d\left(F^{n-1}(x), F^{n}(x)\right)+d\left(x^{*}, F\left(x^{*}\right)\right)\right\} \\
& =\frac{1}{2} d\left(F^{n-1}(x), F^{n}(x)\right) \\
& \leq \frac{1}{2}\left\{d\left(F^{n-1}(x), x^{*}\right)+d\left(F^{n}(x), x^{*}\right)\right\} .
\end{aligned}
$$

That is,

$$
d\left(F^{n}(x), x^{*}\right) \leq d\left(F^{n-1}(x), x^{*}\right) .
$$

Thus $\left\{\mathrm{d}\left(\mathrm{F}^{\mathrm{n}}(\mathrm{x}), \mathrm{x}^{*}\right)\right\}$ is a nonincreasing sequence and is therefore convergent. Since $\mathrm{F}^{\mathrm{n}_{\mathrm{i}}}(\mathrm{x}) \rightarrow z$, we have $\left\{\mathrm{d}\left(\mathrm{F}^{\mathrm{n}_{\mathrm{i}}}(\mathrm{x}), \mathrm{x}^{*}\right)\right\} \rightarrow \mathrm{d}\left(z, x^{*}\right)$ and

$$
d\left(F^{n_{i}+1}(x), x^{*}\right) \leq d\left(F^{n_{i}+1}(x), F(z)\right)+d\left(F(z), x^{*}\right) .
$$

We claim that $z=x^{*}$. If not, then by the continuity of $F$ and $F^{n_{i}}(x) \rightarrow z$, we have

$$
\begin{aligned}
d\left(z, x^{*}\right) & =\lim _{i \rightarrow \infty} d\left(F^{n_{i}}(x), x^{*}\right)=\lim _{n \rightarrow \infty} d\left(F^{n}(x), x^{*}\right) \\
& =\lim _{i \rightarrow \infty} d\left(F^{n_{i}+1}(x), x^{*}\right) \leq d\left(F(z), x^{*}\right),
\end{aligned}
$$


a contradiction to (5).

Hence

$$
\lim _{n \rightarrow \infty} d\left(F^{n}(x), x^{*}\right)=\lim _{i \rightarrow \infty} d\left(F^{n_{i}}(x), x^{*}\right)=\lim _{i \rightarrow \infty} d\left(F^{n_{i}}(x), z\right)=0 .
$$

This completes the proof.

Next we give our convergence result for a Prešić-Kannan nonexpansive mapping on the product of convex metric spaces.

Theorem 7 Let $\mathrm{X}$ be a convex metric space with continuous convex structure $\mathrm{W}$ and $\mathrm{C}$ be a nonempty bounded closed and convex subset of $\mathrm{X}$. Let $\mathrm{f}: \mathrm{C}^{\mathrm{k}} \rightarrow$ $\mathrm{C}$ be a continuous Prešić Kannan nonexpansive mapping with a fixed point $x^{*}$, that is, $f\left(x^{*}, x^{*}, \ldots, x^{*}\right)=x^{*}$. Set $F_{\lambda}(x)=W(x, f(x, x, \ldots, x), \lambda)$ for some $\lambda \in(0,1)$ and let $\mathrm{d}\left(\mathrm{F}_{\lambda}(\mathrm{x}), \mathrm{x}^{*}\right)<\mathrm{d}\left(\mathrm{x}, \mathrm{x}^{*}\right)$ for $\mathrm{x} \neq \mathrm{x}^{*}$. Generate $\left\{\mathrm{x}_{\mathrm{n}}\right\}$ by: $x_{1} \in C, x_{n+1}=F_{\lambda}\left(x_{n}\right) \supset F_{\lambda}\left(x_{n_{i}}\right)=x_{n_{i}+1} \rightarrow z$. Then $z=x^{*}$ and $x_{n} \rightarrow x^{*}$.

Proof. Note that $x^{*}$ is a fixed point of $F_{\lambda}$ as $F_{\lambda}\left(x^{*}\right)=W\left(x^{*}, f\left(x^{*}, x^{*}, \ldots, x^{*}\right), \lambda\right)=$ $W\left(x^{*}, x^{*}, \lambda\right)=x^{*}$.

Define $\mathrm{F}: \mathrm{C} \rightarrow \mathrm{C}$ by

$$
\mathrm{F}(z)=\mathrm{f}(z, z, \ldots, z) \text { for all } z \in \mathrm{C} .
$$

As calculated in Theorem 5, $\mathrm{F}$ is a Kannan nonexpansive mapping.

Since

$$
\begin{aligned}
d\left(F\left(x_{n}\right), x^{*}\right) & =d\left(F\left(x_{n}\right), F\left(x^{*}\right)\right) \\
& \leq \frac{1}{2}\left\{d\left(x_{n}, F\left(x_{n}\right)\right)+d\left(x^{*}, F\left(x^{*}\right)\right)\right\} \\
& =\frac{1}{2} d\left(x_{n}, F\left(x_{n}\right)\right) \\
& \leq \frac{1}{2}\left\{d\left(x_{n}, x^{*}\right)+d\left(F\left(x_{n}\right), x^{*}\right)\right\}
\end{aligned}
$$

we get

$$
d\left(F\left(x_{n}\right), x^{*}\right) \leq d\left(x_{n}, x^{*}\right) .
$$

Therefore

$$
\begin{aligned}
d\left(x_{n+1}, x^{*}\right) & =d\left(F_{\lambda}\left(x_{n}\right), x^{*}\right) \\
& =d\left(W\left(x_{n}, F\left(x_{n}\right), \lambda\right), x^{*}\right) \\
& \leq \lambda d\left(x_{n}, x^{*}\right)+(1-\lambda) d\left(F\left(x_{n}\right), x^{*}\right) \\
& \leq \lambda d\left(x_{n}, x^{*}\right)+(1-\lambda) d\left(x_{n}, x^{*}\right) \\
& =d\left(x_{n}, x^{*}\right) .
\end{aligned}
$$


This gives that $\left\{d\left(x_{n}, x^{*}\right)\right\}$ is a nonincreasing sequence and is therefore convergent.

Also

$$
d\left(x_{n_{i}+1}, x^{*}\right) \leq d\left(x_{n_{i}+1}, F_{\lambda}(z)\right)+d\left(F_{\lambda}(z), x^{*}\right) .
$$

Since $F_{\lambda}$ is continuous and $x_{n_{i}+1} \rightarrow z$,

$$
\lim _{i \rightarrow \infty} d\left(x_{n_{i}+1}, x^{*}\right) \leq d\left(F_{\lambda}(z), x^{*}\right) .
$$

Hence

$$
d\left(z, x^{*}\right)=\lim _{i \rightarrow \infty} d\left(x_{n_{i}+1}, x^{*}\right) \leq d\left(F_{\lambda}(z), x^{*}\right) .
$$

By the given fact that $d\left(F_{\lambda}(x), x^{*}\right)<d\left(x, x^{*}\right)$ for $x \neq x^{*}$, we conclude that $z=\chi^{*}$. This completes the proof.

Next, we approximate fixed point of a Prešić-Kannan nonexpansive mapping by using Mann iterations [14] in the product of uniformly convex metric spaces. The following lemma is crucial to prove our next theorem.

Lemma 1 [22] Let $\mathrm{X}$ be a uniformly convex metric space satisfying Property $(\mathrm{H})$. Then for $\varepsilon>0$ and $r>0$, there exists $\alpha(\varepsilon)>0$ such that

$$
\mathrm{d}(\mathrm{W}(\mathrm{x}, \mathrm{y}, \mathrm{c}), z) \leq \mathrm{r}(1-2 \min \{\mathrm{c}, 1-\mathrm{c}\} \alpha(\varepsilon))
$$

for all $x, y, z \in X, d(x, z) \leq r, d(y, z) \leq r, d(x, y) \geq r \varepsilon$ and $c \in[0,1]$.

Theorem 8 Let $\mathrm{C}$ be a nonempty, bounded, closed and convex subset of a complete uniformly convex metric space $\mathrm{X}$ satisfying Property $(\mathrm{H})$. If $\left\{\boldsymbol{c}_{n}\right\}$ is a sequence in $(0,1)$ such that $\liminf _{\mathrm{n} \rightarrow \infty} \mathrm{c}_{\mathrm{n}}\left(1-\mathrm{c}_{\mathrm{n}}\right)>0$ and $\mathrm{f}$ is a continuous Prešic Kannan nonexpansive mapping from $\mathrm{C}^{\mathrm{k}}$ onto a compact subset of $\mathrm{C}$, then the sequence $\left\{x_{n}\right\}$ defined by:

$$
x_{1} \in C, x_{n+1}=W\left(x_{n}, f\left(x_{n}, x_{n}, \ldots, x_{n}\right), c_{n}\right),
$$

converges to a unique fixed point $x^{*}$ of $\mathrm{f}$.

Proof. As shown in Theorem 5, the mapping F : C $\rightarrow$ C defined by

$$
\mathrm{F}(z)=\mathrm{f}(z, z, \ldots, z) \text { for all } z \in \mathrm{C}
$$

is Kannan nonexpansive. Further choosing $\alpha_{1}=\alpha_{4}=\alpha_{5}=0, \alpha_{2}=\frac{1}{2}=\alpha_{3}$ in Theorem 1, we see that $F$ has a unique fixed point $x^{*} \in C$. As calculated in Theorem $7, \mathrm{~T}$ satisfy the inequality

$$
d\left(F x_{n}, x^{*}\right) \leq d\left(x_{n}, x^{*}\right) .
$$


Therefore

$$
\begin{aligned}
\mathrm{d}\left(x_{n+1}, x^{*}\right) & =d\left(W\left(x_{n}, F\left(x_{n}\right), c_{n}\right), x^{*}\right) \\
& \leq c_{n} d\left(x_{n}, x^{*}\right)+c_{n} d\left(F\left(x_{n}\right), x^{*}\right) \\
& =c_{n} d\left(x_{n}, x^{*}\right)+c_{n} d\left(F\left(x_{n}\right), x^{*}\right) \\
& \leq d\left(x_{n}, x^{*}\right) .
\end{aligned}
$$

This gives that $\left\{d\left(x_{n}, x^{*}\right)\right\}$ is a nonincreasing sequence and is therefore convergent. Let $\lim _{n \rightarrow \infty} d\left(x_{n}, x^{*}\right)=c$ (say). Since $\left\{d\left(x_{n}, F x_{n}\right)\right\}$ is bounded, therefore $\inf _{n \geq 1} d\left(x_{n}, F x_{n}\right)$ exists. We claim that $\inf _{n \geq 1} d\left(x_{n}, F x_{n}\right)=0$. Assume that $\inf _{n \geq 1} d\left(x_{n}, T x_{n}\right)=\sigma>0$. Then

$$
d\left(x_{n}, T x_{n}\right)=d\left(x_{n}, x^{*}\right) \cdot \frac{\sigma}{d\left(x_{n}, x^{*}\right)} \geq d\left(x_{n}, x^{*}\right) \cdot \frac{\sigma}{d\left(x_{1}, x^{*}\right)} .
$$

Hence by Lemma 1, there exists $\alpha\left(\frac{\sigma}{\mathrm{d}\left(\mathrm{x}_{1}, \mathrm{x}^{*}\right)}\right)>0$ such that

$$
\begin{aligned}
d\left(x_{n+1}, x^{*}\right) & =d\left(W\left(x_{n}, T\left(x_{n}\right), c_{n}\right), x^{*}\right) \\
& \leq d\left(x_{n}, x^{*}\right)\left(1-2 \min \left\{c_{n}, 1-c_{n}\right\} \alpha\left(\frac{\sigma}{d\left(x_{1}, x^{*}\right)}\right)\right) .
\end{aligned}
$$

That is

$$
2 c_{n}\left(1-c_{n}\right) \alpha\left(\frac{\sigma}{d\left(x_{1}, x^{*}\right)}\right) \leq d\left(x_{n+1}, x^{*}\right)-d\left(x_{n}, x^{*}\right) .
$$

By $\lim \inf _{\mathrm{n} \rightarrow \infty}$ on both sides in (6), we get that

$$
\alpha\left(\frac{\sigma}{d\left(x_{1}, x^{*}\right)}\right) \liminf _{n \rightarrow \infty} c_{n}\left(1-c_{n}\right)=0 .
$$

But $\liminf _{n \rightarrow \infty} c_{n}\left(1-c_{n}\right)>0$ implies that $\alpha\left(\frac{\sigma}{d\left(x_{1}, x^{*}\right)}\right)=0$, a contradiction. Therefore $\inf _{n \geq 1} d\left(x_{n}, T x_{n}\right)=0$. Then there exists a subsequence $\left\{x_{n_{i}}\right\}$ such that $\lim _{i \rightarrow \infty} d\left(x_{n i}, F\left(x_{n_{i}}\right)\right)=0$. Since $F$ maps $C$ into a compact subset of $C$, this implies that there exists a subsequence $\left\{x_{n_{i_{j}}}\right\}$ of $\left\{x_{n_{i}}\right\}$ such that $\left\{F\left(x_{n_{i_{j}}}\right)\right\}$ converges to a point $u$ in $C$ as $j \rightarrow \infty$. Since $\lim _{j \rightarrow \infty} d\left(x_{n_{i_{j}}}, F\left(x_{n_{i_{j}}}\right)\right)=0$ we obtain that $\lim _{j \rightarrow \infty} x_{n_{i_{j}}}=u=\lim _{j \rightarrow \infty} F\left(x_{n_{i_{j}}}\right)$. But $F$ is continuous, so $u=F(u)$. Since

$$
d\left(u, x^{*}\right)=d\left(F(u), F\left(x^{*}\right)\right) \leq \frac{1}{2}\left\{d(u, F(u))+d\left(x^{*}, F\left(x^{*}\right)\right)\right\}=0,
$$

$\lim _{j \rightarrow \infty} x_{n_{i_{j}}}=u, \lim _{n \rightarrow \infty} d\left(x_{n}, x^{*}\right)$ exists, therefore $x_{n} \rightarrow x^{*}$. 


\section{Acknowledgments}

The author(s) would like to acknowledge the support provided by the Deanship of Scientific Research(DSR) at King Fahd University of Petroleum \& Minerals (KFUPM) for funding this work through project No. IN151014

\section{References}

[1] D. E. Alspach, A fixed point free nonexpansive map, Proc. Amer. Math. Soc., 82 (1981), 423-424.

[2] V. Berinde, V. and M. Păcurar, An iterative method for approximating fixed points of Prešić nonexpansive mappings, Rev. Anal. Numér. Théor. Approx., 38 (2) (2009), 144-153.

[3] V. Berinde, A. R. Khan, M. Păcurar, Coupled solutions for a bivariate weakly nonexpansive operator by iterations Fixed Point Theory Appl. 2014, 2014:149, 12 pp.

[4] M. Bridson and A. Haefliger, Metric spaces of non-positive curvature, Springer-Verlag, Berlin, Heidelberg, New York, 1999.

[5] H. Busemann, Spaces with non-positive curvature, Acta Math., 80 (1948), 259-310.

[6] L. B. Cirić and S. B. Prešić, On Prešić type generalization of the Banach contraction mapping principle, Acta Math. Univ. Comenianae, 76 (2) (2007), 143-147.

[7] H. Fukhar-ud-din, A. R. Khan, Z. Akhtar, Fixed point results for a generalized nonexpansive map in uniformly convex metric spaces, Nonlinear Analysis, 75 (2012) 4747-4760.

[8] H. Fukhar-ud-din, V. Berinde and A. R. Khan, Fixed point approximation of Prešić nonexpansive mappings in product of CAT(0) spaces, Carpatheian J. Math., 32 (3) (2016), 315-322.

[9] R. Kannan, Some results on fixed points-IV, Fund. Math. LXXIV (1972), 181-187.

[10] R. Kannan, Fixed point theorems in reflexive Banach spaces, Proc. Amer. Math. Soc., 38 (1973), 111-118. 
[11] A. R. Khan, M. A. Khamsi and H. Fukhar-ud-din, Strong convergence of a general iteration scheme in CAT(0)-spaces, Nonlinear Anal., 74 (2011), $783-791$.

[12] W. A. Kirk, Geodesic geometry and fixed point theory II, in: Proceedings of the International Conference in Fixed Point Theory and Applications, Valencia, Spain, 2003, pp. 113-142.

[13] M. A. Khamsi and W. A. Kirk, An Introduction to Metric Spaces and Fixed Point Theory, Pure and Applied Mathematics. Wiley-Interscience, New York, 2001.

[14] W. R. Mann, Mean value methods in iterations, Proc. Amer. Math. Soc., 4 (1953), 506-510.

[15] M. Păcurar, Iterative Methods for Fixed Point Approximation, PhD Thesis, "Babeş-Bolyai" University, Cluj-Napoca, 2009.

[16] — A multi-step iterative method for approximating fixed points of Prešić-Kannan operators, Acta Math. Univ. Comenianae, Vol. LXXIX, 1 (2010), 77-88.

[17] — Approximating common fixed points of Prešić-Kannan type operators by a multi-step iterative method, An. Şt. Univ. Ovidius Constan c ta, 17 (1) (2009), pp. 153168.

[18] — A multi-step iterative method for approximating common fixed points of Prešić-Rus type operators on metric spaces, Studia Univ. BabeşBolyai Math., 551 (2010), 149-162.

[19] W. Phuengrattana and S. Suantai, Strong convergence theorems for a countable family of nonexpansive mappings in convex metric spaces, Abstract Applied Anal., vol. 2011, Article ID 929037, 18 pages.

[20] S. B. Prešić, Sur une classe d' inéquations aux différences finites et sur la convergence de certaines suites, Publ. Inst. Math. (Beograd)(N.S.), 5 (19) (1965), 75-78.

[21] I. Rus,An iterative method for the solution of the equation $x=$ $\mathrm{f}(\mathrm{x}, \mathrm{x}, \ldots, \mathrm{x})$, Rev. Anal. Numer. Theor. Approx., 10 (1) (1981), 95-100.

[22] T. Shimizu, A convergence theorem to common fixed points of families of nonexpansive mappings in convex metric spaces, in Proceedings of the 
International Conference on Nonlinear and Convex Analysis (2005), 575585 .

[23] T. Shimizu and W. Takahashi, Fixed points of multivalued mappings in certain convex metric spaces, Topol. Methods Nonlinear Anal., 8 (1996), 197-203.

[24] P. M. Soardi, Struttura quasi normale e teoremi di punto unito, Rend. Istit. Mat. Univ. Trieste, 4 (1972), 105-114.

[25] W. Takahashi, A convexity in metric spaces and non-expansive mappings, Kodai Math. Sem. Rep., 22 (1970), 142-149.

Received: February 21, 2017 